\title{
Application of Lidar Techniques to Time-of-Flight Range Imaging
}

\author{
Refael Whyte ${ }^{1}$, Lee Streeter ${ }^{1, *}$, Michael J. Cree ${ }^{1}$, and Adrian A. Dorrington ${ }^{1}$ \\ ${ }^{1}$ School of Engineering, The University of Waikato, Private Bag 3105, Hamilton 3240, New Zealand \\ *Corresponding author: Ivs2@phys.waikato.ac.nz
}

Compiled October 14, 2015

\begin{abstract}
Amplitude modulated continuous wave (AMCW) time-of-flight (ToF) range imaging cameras measure distance by illuminating the scene with amplitude modulated light and measuring the phase difference between the transmitted and reflected modulation envelope. This method of optical range measurement suffers from errors caused by multiple propagation paths, motion, phase wrapping and non-ideal amplitude modulation. In this paper a ToF camera is modified to operate in modes analogous to continuous wave (CW) and stepped frequency continuous wave (SFCW) lidar. In CW operation the velocity of objects can be measured. CW measurement of velocity was linear with true velocity $\left(R^{2}=0.9969\right)$. Qualitative analysis of a complex scene confirms that range measured by SFCW is resilient to errors caused by multiple propagation paths, phase wrapping and non-ideal amplitude modulation which plague AMCW operation. In viewing a complicated scene through a translucent sheet, quantitative comparison of AMCW with SFCW demonstrated a reduction in the median error from $-1.3 \mathbf{m}$ to $-0.06 \mathbf{m}$ with inter-quartile range of error reduced from $4.0 \mathrm{~m}$ to $0.18 \mathrm{~m}$. () 2015 Optical Society of America
\end{abstract}

OCIS codes: (110.6880) Three-dimensional image acquisition; (150.5670) Range finding; (280.3640) Lidar; (150.6910) Threedimensional sensing

http://dx.doi.org/10.1364/ao.XX.XXXXXX

\section{INTRODUCTION}

Time-of-flight (ToF) imaging based optical metrology has grown significantly in recent years [1-10]. As the capability of ToF cameras continues to improve more advanced range imaging modalities become increasingly feasible, promising better measurement. A common current method of video frame rate ToF range imaging is amplitude modulating the light source and indirectly measuring the time-of-flight as the phase difference between the emitted and reflected light. This technique is known as homodyne amplitude modulated continuous wave (AMCW) ToF range imaging [1]. A key improvement of AMCW ToF range cameras in recent years has been the modulation bandwidth from $20 \mathrm{MHz}$ up to $150 \mathrm{MHz}$ [3, 4]. This increase in bandwidth opens the door to new multi-frequency operating modes, including techniques previously restricted to other hardware, that enhance the operation of ToF camera systems. Methods from single point lidar systems which have focused on accurate and precise range measurements, can now receive renewed attention in application to ToF camera operation. In particular frequency modulated continuous wave (FMCW) schemes [2] from laser based lidar systems achieve high range resolution in the order of micrometres, with large range windows of the order of me- tres, and thus are appealing alternative operation methods for AMCW ToF camera hardware.

Measurement errors inherent to AMCW ToF range camera operation has limited the achievable accuracy of the range measurements. The major causes of measurement errors [11] are multiple propagation paths from the light source to pixel, commonly called multi-path interference [1], phase wrapping of the modulation envelope from $2 \pi$ back to 0 [6], aliasing of higher order harmonics in the modulation signal that cause nonlinearity in the distance measurement $[5,12]$, and motion artefacts arising due to the multi-frame operation of ToF [7].

Homodyne AMCW distance measurements are negatively affected by motion as the method traditionally employed to compute depth does not take into account motion. Both coded exposure [7] and optical flow [8] methods for motion correction that come from computer vision have been applied to ToF range cameras, but still treat motion as an artefact to be corrected. Recently heterodyne AMCW (light source and sensor modulated at different frequencies) was applied to the problem of radial velocity measurement [13].

Homodyne AMCW has been the dominant operating mode of indirect ToF range cameras due to its ease of implementation and low computational overhead. However due to the 
measurement errors inherent in homodyne AMCW operation other operating modes are becoming more attractive. Homodyne AMCW is just one modulation scheme for ToF imaging, and other schemes have previously been employed to try and resolve these measurement errors. During early efforts into ToF range imaging Dorrington et al. [9] investigated Heterodyne AMCW. Heterodyne ToF operates by modulating the light source and sensor at slightly different modulation frequencies. Heterodyne has an advantage over homodyne AMCW for increased resilience to higher order harmonics in the modulation signal. However, practical difficulties in locking the relative modulation frequencies and phase of the light source and sensor have limited the applications of heterodyne ToF. While the traditional AMCW ToF range cameras operation use a single static modulation frequency, the ability to drive the hardware and different modulation frequencies has lead to research into multi-frequency operation.

As early as 2002, Gulden et al. [10] investigated stepped frequency continuous wave (SFCW) radar modulation in ToF to resolve objects at different distances. However, they were limited by the modulation bandwidth of the camera hardware available then. Godbaz et al. [1] used measurements at two modulation frequencies and a lookup table to resolve multi-path interference. Freedman et al. [14] expanded Godbaz's work to three modulation frequencies and used a compressed lookup table. Kirmani et al. [15] measured five modulation frequencies and used spectrum estimation techniques to resolve the multi-path interference and perform phase unwrapping. Droeschel et al. [16] also employed multi-frequency operation of AMCW to perform phase unwrapping. Most recently the computational imaging community has progressed the concept of multifrequency ToF, acquiring range data from tens to as many as one-hundred or more frequencies and employing sparse deconvolution to separate multiple returns [17], perform transient imaging [18] (using hundreds of frequencies) and imaging objects in scattering media [3]. Dorrington et al. [19] used two modulation frequencies, one double the other, to resolve two propagation paths. Bhandari et al. [20] used sparse deconvolution and multiple complex measurements to resolve $K$ propagation paths. Fourier analysis on the multifrequency measurements for transient imaging was done by Lin et al. [21]. The research trend has been to use increasingly more frequencies to solve the multi-path problem, with increasing success. However, more frequencies means more measurement over a longer data acquisition period than standard ToF operation. This motivates the search for alternative ToF camera operation that is resilient to error and can run at standard video rates. Multi-frequency operation of ToF hardware is progressively resembling radar operation, and the expanding bandwidth capabilities of the hardware are making the prospective use of radar like techniques an increasingly practical possibility.

Continuous wave $(\mathrm{CW})$ radars have been employed since the Second World War, and there is vast literature on their design and applications [22]. CW lidar devices have been used for solid state velocity measurements [23]. Frequency modulated continuous wave (FMCW) devices measure distance by emitting a sweep of frequencies. The reflected signal is mixed (in this context mixing means multiplication of two periodic signals to produce a new signal with a new frequency [22], an engineered process distinct from mixed-pixel effect) with the transmitted signal and the difference in frequency encodes the distance travelled. FMCW is commonly used in radar systems [24], and in lidar systems for high resolution range measurements [2]. A slight modification to FMCW is stepped frequency continuous wave (SFCW) radar, In which a single frequency is emitted at a time, with an increase in frequency for each step. SFCW is common in ground penetrating radar where the signal to noise ratio (SNR) is important and there are more relaxed measurement times [24], and SFCW has been demonstrated in lidar by Simpson et al. [25].

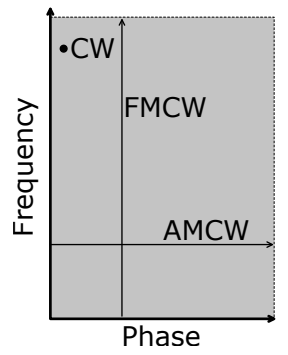

Fig. 1. Diagram illustrating the relationship between time-offlight modalities considered. (CW is continuous wave and is at a fixed frequency and phase, AMCW is amplitude modulated continuous wave and involves varying the phase and FMCW is frequency modulated continuous wave and involves varying the frequency.)

The relationship between the modalities from radar that we consider for ToF are illustrated in Fig. 1. In continuous wave $(\mathrm{CW})$ the phase and the frequency are held constant and velocity is measured from the Doppler shift in frequency. In AMCW the phase is varied and in FMCW the frequency is varied, both to measure range. Each modality can be implemented in a ToF system, either radar or lidar. The advantage of full-field range imaging technology over single point lidar is the efficiency and low cost with which a large volume of data is provided by the imaging system, albeit at a lower precision.

In this paper we modify the operation of a ToF range camera to perform the radar techniques of continuous wave $(\mathrm{CW})$ to measure velocity along the optical axis, and stepped frequency continuous wave (SFCW) (a variant of FMCW suitable for ToF camera hardware) ranging to investigate resilience to measurement errors. Intensity modulated SFCW lidar has been employed by Simpson et al. [25] for resolving multiple translucent sheets, and we show that SFCW can be applied to ToF range cameras for full field video frame rate range cameras. Standard lidar processing algorithms are applied to estimate the velocity of moving objects and the distance of stationary objects. The advantages and disadvantages of operating a ToF camera in CW and SFCW modes are evaluated.

\section{TIME-OF-FLIGHT BACKGROUND}

Current ToF range cameras operate by illuminating the scene with amplitude modulated light. The light travels the distance $2 d$, where $d$ is the range from the camera to the scene, and on receipt at the camera sensor the time-of-flight induces a phase shift $\phi$ in the amplitude modulation of

$$
\phi(\omega, d)=\frac{2 \omega d}{c},
$$

where $c$ is the speed of light in the medium and $\phi$ is a function of $\omega$, the modulation angular frequency, and of $d$. Note that in the literature on full-field range imaging it is typical to take $\phi$ constant, but we shall consider $\phi$ a function of time if either the 
modulation frequency is changing (as then $\omega=\omega(t)$ ) or if there is motion in the scene (as then $d=d(t)$ ).

In the idealised case only the fundamental harmonic is present in the modulation [5] thus the received signal at a pixel of the sensor is given by

$$
s(t)=\alpha(t) \sin (\omega t+\phi(\omega, d))+\beta,
$$

where $\alpha$ is the amplitude of the reflected light (and possibly a function of time) and $\beta$ is the background light. The aim is to efficiently recover $\phi$ (thus the range $d$ and possibly the velocity $v$ ) and the amplitude of modulation $\alpha(t)$ from measurements in time of the received radiation at each pixel of the sensor.

The received light is mixed (multiplied) at the sensor during the $j$-th raw frame capture with a reference signal, $g_{j}(t)$ given by,

$$
g_{j}(t)=\frac{1}{2} \sin \left(\omega t+\tau_{j}\right)+\frac{1}{2},
$$

where $\tau_{j}$ is an adjustable extra phase shift relative to the emitted illumination added to the reference signal and kept constant during each raw frame capture. The factor of a half expresses the ideal maximum modulation depth of the sensor [26].

A single frame capture of integration period $T$, where $T$ is sufficiently large compared to the period of the modulation frequency, results in,

$$
\begin{aligned}
h_{j}(\omega, \tau ; d) & =\int_{0}^{T} s(t) g_{j}(t) d t \\
& =\frac{1}{2} \int_{0}^{T} \alpha(t) \cos \left(\phi(\omega, d)-\tau_{j}\right) d t+b \\
& =a_{j} \cos \left(\phi_{j}-\tau_{j}\right)+b,
\end{aligned}
$$

where the $a_{j}$ and $b$ encode the amplitude and background component. In the last line it is assumed that any motion in the scene is sufficiently slow that $\alpha(t)$ and $d(t)$ can be considered constant during the frame integration, namely that $a(t) \approx a_{j}$ and $d(t) \approx d_{j}$ for the $j$-th frame capture. In addition the modulation frequency is never changed during frame capture, thus $\phi(\omega, d) \approx \phi_{j}$.

The analysis, so far, is general enough to encompass all three ranging modalities considered herein. In the following further restrictions are applied to derive in turn, CW, AMCW (the typically used ranging modality) and SFCW.

\section{A. Continuous Wave Measurement of Velocity}

Continuous wave (CW) measurement devices [22], radar and lidar, emit electromagnetic signals at a single frequency and phase. If the target is stationary then the reflected frequency is the same as transmitted. If the object is moving its velocity component in the direction of signal propagation is encoded by a Doppler shift in the reflected signal's frequency.

Let the motion in the scene be constrained to be constant velocity linear motion directly towards or away from the sensor, thus

$$
d=v t+d_{0},
$$

where $v$ is the velocity of the point being imaged and $d_{0}$ is its distance at $t=0$. With the assumption of sufficiently slow motion the distance during the $j$-th capture can be taken to be,

$$
d_{j} \approx v t_{j}+d_{0}
$$

where $t_{j}=j \Delta t$ for a frame repetition time of $\Delta t$. Hence

$$
\phi_{j}=\frac{2 \omega d_{j}}{c}=\frac{2 \omega\left(v t_{j}+d_{0}\right)}{c} .
$$

It should also be noted that as an object moves closer or more distant from the sensor the amount of light received back changes. The amplitude is a function of the distance, reflectively of the imaged surface $\Gamma$, and surface orientation, indeed,

$$
\alpha=\Gamma \frac{\mathbf{n} \cdot \mathbf{1}}{d^{2}},
$$

where $\mathbf{n}$ is the normal to the imaged surface patch and $\mathbf{l}$ is a unit vector representing the source light direction on to the imaged surface patch. Assuming the surface orientation stays constant during the object's motion (which is approximately true for slow to moderate speed and a high frame capture rate), then the amplitude changes as,

$$
\alpha_{j}=\frac{a_{j} d_{0}^{2}}{\left(v t_{j}+d_{0}\right)^{2}} .
$$

Inserting into Eqn. 6 gives the measured signal as,

$$
h_{j}=a_{j} \cos \left(\frac{2 \omega\left(v t_{j}+d_{0}\right)}{c}-\tau_{j}\right)+b .
$$

Note that the velocity of the object is encoded in the frequency of oscillation of the received signal (the conventional Doppler shift), thus can be recovered by spectral analysis of the received signal if the frame captures $h_{j}$ are evenly spaced in time and if $\tau_{j}$ is set to an unchanging value. It is most convenient to choose $\tau_{j}=0$.

This mode of imaging to recover velocity is analogous to continuous wave $(\mathrm{CW})$ Doppler lidar. As no extra phase offsets $\left(\tau_{j}\right)$ are introduced into the reference signal, and as the modulation frequency $(\omega)$ is fixed, this mode of operation is a single point on the diagram of Figure 1. Because of this $\mathrm{CW}$ only recovers the velocity, not the range. $\mathrm{CW}$ devices are easy to manufacture and as the signal is continuous they have good SNR, thus can accurately measure the velocity of objects.

To estimate the frequency, hence the velocity, either zero crossing spacing or spectrum estimation techniques from $\mathrm{CW}$ Doppler radar may be employed [22]. Herein we use the zero crossing method.

\section{B. Amplitude Modulated Continuous Wave}

One (or both) of the phase and the modulation frequency must be varied to recover the range to the scene. It is convenient to first discuss the AMCW mode of operation as it is the only technique (with very few exceptions) applied in full-field range imaging. In AMCW it is the phase of the reference signal that is adjusted between raw frame captures (cf. Figure 1). The important advantage of AMCW is that it can be efficiently implemented on sensors that have a limited modulation bandwidth, a characteristic of almost all full-field range imaging technology to date.

Let the phase of the reference signal $g_{j}$ be adjusted by a fixed increment each frame capture for a total increment of $2 \pi$ over $N$ captures, that is, let

$$
\tau_{j}=\frac{2 \pi j}{N}, \quad j=0,1, \ldots, N-1 .
$$

Also assume a static scene, namely that $a_{j}=a$ and $\phi_{j}=\phi$ for all frame captures, then the captured signal is

$$
h_{j}=a \cos \left(\phi-\frac{2 \pi j}{N}\right)+b,
$$


thus by capturing at least three frames, the amplitude $a$, phase $\phi$ and offset $b$ of the signal can be recovered by discrete Fourier transform (DFT). The range $d$ to the scene is then found from $\phi$ by Eqn. 1. Even though a minimum of three frame captures is required, it is common to use four [27], and there are extant implementations that use even more frames [5] for reasons discussed below.

As the fundamental frequency bin of the DFT demodulates the measured $h_{j}$ [1], it can be convenient to represent the recovered amplitude and phase as a complex number $\zeta$ by

$$
\zeta=a e^{-i \phi}
$$

where $i$ is the imaginary unit.

Despite the ubiquity of AMCW in full-field range imaging, AMCW reconstructed range measurements suffer from a number of very significant deleterious error sources $[5,28]$, namely wrapping of the recovered phase (thus range) from $2 \pi$ back to 0 , multiple propagation paths from the light source to the camera pixel, and ambiguous aliasing of higher order harmonics in the correlation waveform signal.

The range at which the phase wrapping occurs is called the ambiguity distance $d_{a}$ and is easily calculated from Eqn. 1 by setting $\phi=2 \pi$. Objects that are more distant than the ambiguity distance are actually measured to be in the range $\left[0, d_{a}\right)$ thus are measured to be closer than they actually are by an amount that is an integer multiple of the ambiguity distance. There are two broad techniques for phase unwrapping in ToF cameras: one is to use the spatial information from the surrounding pixels in the sensor [6], and the other takes measurements at multiple (often two) modulation frequencies [28]. Using multiple modulation frequencies borrows phase unwrapping techniques from phase modulation interferometry.

ToF hardware is typically implemented with digital logic thus the modulation signals $s(t)$ and $g(t)$ are in reality (approximate) square waves, thus higher order harmonics are present in the measured correlation function $h_{j}$. If the sampling rate (i.e. the number of samples $N$ ) of the correlation signal does not satisfy the Shannon sampling criterion then ambiguous aliasing of higher order harmonics occurs, and if the aliases are at the fundamental (which encodes the range) substantial measurement errors can occur. For example, if $N=4$ (a common choice) then the third and all higher order odd harmonics have aliases at the fundamental, thus corrupt the range measurement. The obvious solution is to increase the number of captured frames $N$, but that increases the time of a full range recovery and to achieve real time acquisition (video rate) $N$ must be kept small.

For fully known harmonic contamination of the received correlation signal one can calibrate for the error, for instance Lindner et al. [12] review and improve the calibration process by modelling square wave and sine wave modulation together. One can also calibrate by modelling a spectral decomposition of the correlation waveform [29]. Either way, calibration can be unwieldy if the spectral content of the signal changes with environmental factors such as temperature. In a more general approach, Payne et al. [5] demonstrated that by changing the phase of the reference signal during the integration period the higher order harmonics can be cancelled, at the expense of a small tolerable loss in signal to noise ratio (SNR).

Multi-path interference is caused by multiple propagation paths from the light source to the pixel. This is caused by interreflections in the scene and/or the lens-camera system, subsurface scattering and mixed pixels due to the solid angle viewed by a pixel covering two distinct objects. Multi-path interference caused by multiple propagation paths can be described as the closest return plus the integral of all other more distant returns [30]

$$
\zeta=a_{1} e^{-i \phi_{1}}+\int_{\Delta+\phi_{1}}^{\infty} a_{m} e^{-i \phi_{m}} d m
$$

This is often approximated by $M$ sparse returns meaning that each return is physically separated from the others such that [30]

$$
\zeta \approx \sum_{m=1}^{M} a_{m} e^{-i \phi_{m}} .
$$

Note that the combination of phase-shifted sines (multiple returns) is itself a sine function of which only the resultant sine amplitude and phase can be recovered by AMCW. As multiple propagation paths are commonplace in typical scenes, and the corruption is scene dependent and often substantial, multipath interference is the biggest problem to quantitative range measurement with current full-field range imaging technology.

\section{Stepped Frequency Continuous Wave}

The other option to measuring range in a static scene (when $a_{j}=a$ and $d_{j}=d$ ) is to modify the modulation frequency during acquisition. Let the modulation frequency be held constant during any individual frame capture, and be incremented by a constant amount between each capture, that is, stepped frequency continuous wave. The modulation frequency during the $j$-th capture is therefore

$$
\omega_{j}=\omega_{0}+j \Delta \omega
$$

and $\tau_{j}=0$ (for a convenient constant value as there is no need for phase increments in SFCW). The measured correlation signal is

with

$$
h_{j}=a \cos \left(\phi_{j}\right)+b \text {, }
$$

$$
\phi_{j}=\phi\left(\omega_{j}\right)=\frac{2 \omega_{j} d}{c} .
$$

Since $\phi_{j}$ increases linearly with each captured frame of the acquisition the range is encoded as the frequency of the received oscillating signal, thus can be recovered by spectral analysis of the $h_{j}$.

This is classic SFCW modulation for radar [31], in which the frequency of the correlation signal encodes the range. Normally the DFT is taken to compute the frequency, however the bandwidth of a ToF camera is limited compared to modern radar hardware. Therefore other spectral estimation techniques are used, described below in Sec. C.1. The relationship between the estimated frequency $\omega_{\text {est }}$ and the distance is

$$
\omega_{e s t}=\frac{B}{c} \frac{d}{2}
$$

where $B$ is the bandwidth of measured frequencies.

In the following we examine the effect of each of the major forms of error on SFCW. We show that SFCW, unlike AMCW, is only limited by hardware dependent factors, not by light scattering, and that the modulation frequency bandwidth of the camera limits the resolving power of the measured range in the Nyquist sense. Furthermore it is reasonable to expect that the bandwidth of ToF hardware to increase in time, and subsequently the bandwidth limitation to reduce. The forms of error that limit AMCW most severely, especially multi-path interference, are intrinsically resolved by the SFCW modality. 


\section{C.1. Distance Resolving Power}

The resolving power of SFCW devices depends on the accuracy of the frequency estimation. Traditionally the DFT has been employed by the Radar community, however the minimal resolvable distance with a Fourier transform is [22]

$$
\Delta R=\frac{c}{2 B} .
$$

Currently ToF cameras typically operate up to $150 \mathrm{MHz}$, therefore the distance quantization is $1 \mathrm{~m}$. The use of modern spectral estimation techniques allows for better range resolution [32]. As ToF hardware continues to progress it is reasonable to expect the bandwidth to increase and, with it, the depth resolution of SFCW to improve.

There are three main methods of spectral analysis methods, non-parametric methods, parametric methods, and subspacebased estimators. Non-parametric methods make very few hypotheses on the input signal, whereas parametric methods require $a$ priori information about the signal. A special case of parametric methods is that of a signal composed of a finite set of sinusoidal functions, which has applications in signal and image processing, and solved using subspace-based estimators. The process that generates the spectrum is well known, there are likely to be a limited number of frequencies present, and only a few data points are measured. Parametric and subspace based methods are natural solutions to the problem.

\section{C.2. Multi-path Interference}

When multiple propagation paths are present waveforms of different frequency are present in the correlation signal,

$$
h_{j}=\sum_{m=1}^{M} a_{m} \cos \left(\frac{2 \omega_{j} d_{m}}{c}\right) .
$$

The primary return corresponds to $m=1$, which has distance $d_{1}$ that we want to measure. Analogously, diffuse multi-path interference is described as

$$
h_{j}=a_{1} \cos \left(\frac{2 \omega_{j} d_{1}}{c}\right)+\int_{d_{1}+\Delta}^{\infty} a(v) \cos \left(\frac{2 \omega_{j} v}{c}\right) d v
$$

where $v$ is continuous distance from the primary return, and $a(v)$ is a scene dependent continuous brightness function distinct from Eqn. 11. Under Fourier analysis the different propagation paths manifest at different frequencies. Which return corresponds to the direct path is scene dependent and, at this stage, requires user interaction. If the camera bandwidth is adequate, or if the spectral estimation technique can resolve the frequencies, then the direct return can be measured without contamination by multi-path interference.

\section{C.3. Harmonic Aliasing}

Harmonic aliasing is one source of measurement error in AMCW range imaging. Since square waves are used for the modulation signal there are higher order odd harmonics present

$$
h(\omega)=\sum_{k=0}^{\infty} \frac{a}{(2 k+1)^{2}} \cos \left(\frac{2(2 k+1) \omega d}{c}\right) .
$$

The higher order harmonics appears as higher frequencies in the spectrum than the fundamental, therefore (in the absence of multi-path) in choosing the lowest frequency light return, harmonics do not affect the distance estimation.

\section{C.4. Phase Wrapping}

ToF range cameras when operating in AMCW mode suffer from phase wrapping. In imaging a scene of a given range of distances, phase wrapping becomes more of an issue at higher modulation frequencies. Frequency aliasing sets the ambiguity distance in SFCW radars. The ambiguity distance for SFCW is [24]

$$
d_{\text {amg }}=\frac{c}{2 \Delta f}
$$

where $\Delta f$ is the separation between measured frequencies. With SFCW the frequency separation is normally less than the AMCW modulation frequency, therefore SFCW is less likely to encounter ambiguous distances.

\section{EXPERIMENTAL SETUP}

We capture data using a proprietary ToF range camera which has a maximum modulation frequency of $150 \mathrm{MHz}$. We test the CW Doppler velocimetry by moving a flat white foam board with diffuse reflectance under controlled conditions. The white foam board is placed on a $3.0 \mathrm{~m}$ translation stage (Macron Dynamics Inc, Croydon, PA, USA), and the translation stage is moved at varying velocities starting at $0.1 \mathrm{~m} / \mathrm{s}$ going up to $1.1 \mathrm{~m} / \mathrm{s}$ in increment of $0.1 \mathrm{~m} / \mathrm{s}$. The ToF camera is modulated at $150 \mathrm{MHz}$, with each raw frame having an integration period of $0.5 \mathrm{~ms}$ at a frame rate of $25 \mathrm{fps}$. The full field image results are calculated from using a zero crossing detection algorithm.

For the SFCW mode experimentation a scene with a maximum distance of $8 \mathrm{~m}$ is imaged. The scene is comprised of both diffuse and specular objects, and is measured twice using both SFCW and AMCW: the first time without any foreground objects; and the second time thorough a specular translucent sheet placed $1 \mathrm{~m}$ from the camera and angled approximately $30^{\circ}$ to the sensor plane to minimise saturation of the sensor due to specular reflection. The translucent sheet is the size of an A4 sheet of paper and fully covers both the field of view of the camera and the light source.

The purpose of this experiment is to compare the effect of multi-path interference due to the light reflected from the translucent sheet on the ability of AMCW and SFCW to measure the distance to the objects behind it. The scene is measured with a frequency sweep over $5-150 \mathrm{MHz}$ inclusive in $5 \mathrm{MHz}$ increments, and at $20 \mathrm{MHz}$ in AMCW mode with nine phase steps. The choice of nine steps is large enough to minimise harmonic aliasing error [5], but is small enough for a video frame rate of 30 depth images per second in AMCW operation. The modulation frequency of $20 \mathrm{MHz}$ is used because of the relatively long distances imaged over. The SFCW is calibrated for: fixed pattern noise by measurement and subtraction; and likewise for amplitude calibration for change with frequency.

The spectral components due to the light returns are found by the multiple signal classification (MUSIC) method [33]. The MUSIC method is well researched pseudo-spectrum algorithm that computes principle components (PC) of a correlation matrix built from the input signal. More PCs are taken than there are harmonics in the input signal, so the PCs that correspond to the smallest eigenvalues are orthogonal to those harmonics, so inverting the magnitude of the Fourier Transform yields peaks at the frequencies of the harmonics due to the light return. MUSIC was chosen for its ability to resolve harmonics between the bins of the discrete Fourier transform and its robustness to 'false peaks' that may arise from noise. The implementation of MUSIC in the Matlab signal processing toolbox function pmusic.m is 
used with order 5 and length 2048. Two peaks of the MUSIC pseudo-spectrum are taken, corresponding to objects at two different distances. With the a priori knowledge that the translucent sheet is closest, the more distant of the two peaks is chosen from which we find the estimate of the true return. The 30 samples are down sampled for testing the effect of using a different number of measurements.

The accuracy of depth measurements with SFCW is tested over $1.05 \mathrm{~m}$ without multi-path, by placing a white foam board on the translation stage and moving the stage in increments of $0.05 \mathrm{~m}$. At each location the thirty frequencies from $5 \mathrm{MHz}$ up to $150 \mathrm{MHz}$ in increments of $5 \mathrm{MHz}$ are measured, and the depth is computed with the MUSIC method. SFCW depth measurement is compared to AMCW at $50 \mathrm{MHz}$ computed with nine phase steps. One hundred depth measurements are performed so the variance of the depth measurements can be compared.

\section{RESULTS AND DISCUSSION}

\section{A. Continuous Wave Modulation}

The measurements of one-hundred pixels, about the centre of the target object, over time for two different velocities on the translation stage are summarised in Fig. 2. The ambiguity distance at $150 \mathrm{MHz}$ is $1 \mathrm{~m}$, therefore three oscillations are observed, and the decay is due to the reduction in the amplitude as the object moves away from the camera. As expected the faster moving object has higher frequency than the slower object. The estimated velocity is plotted against actual velocity in Fig. 3. An F-test shows that the estimated velocity is linear with the actual $\left(F=2893, \rho=1.33 \times 10^{-12}\right)$, which supports the claim that $\mathrm{CW}$ ToF measures the velocity. The frame rate of $25 \mathrm{fps}$ limits the maximum measurable velocity. The low frame rate is because the ToF camera was not designed to operate in CW mode, but could easily achieve $120 \mathrm{fps}$ if redesigned. The maximum measurable frequency is set by the integration period of the pixel and its modulation frequency. With the current settings of an integration period of $0.5 \mathrm{~ms}$ at a modulation frequency of $150 \mathrm{MHz}$ the maximum velocity measurable is $200 \mathrm{~m} / \mathrm{s}$. Which is much faster than common applications of ToF applications will encounter, such as computer human interaction.

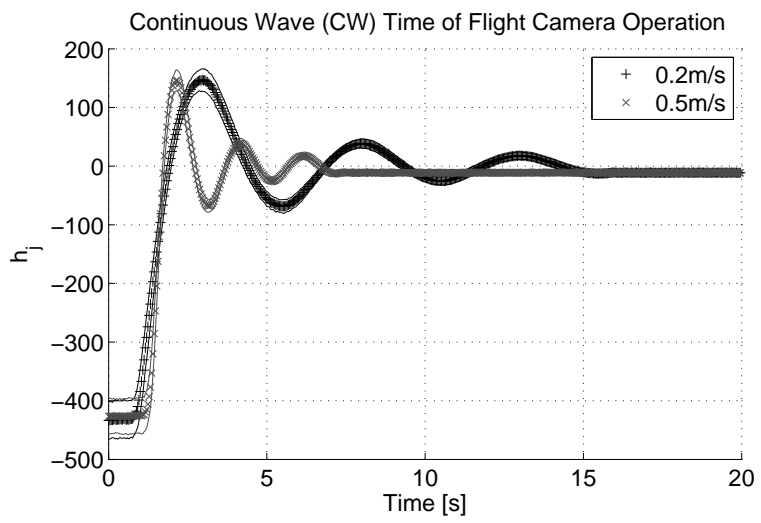

Fig. 2. Measurements in time for an object moving $3 \mathrm{~m}$ on a translation stage at different speeds. Markers are means and solid lines indicate three standard deviations.

An example of the full field results are plotted in Figs. 4 and 5. In Fig. 4 the cardboard sheet is moving at different velocities, with the velocity image is taken when the cardboard sheet is the
Table 1. Comparison between translation stage velocity and the measured for a region of 10878 pixels.

\begin{tabular}{l|ll}
$\begin{array}{l}\text { Translation Stage } \\
\text { Velocity } \mathbf{m} / \mathbf{s}\end{array}$ & $\begin{array}{l}\text { Measured } \\
\text { Mean } \mathbf{m} / \mathbf{s}\end{array}$ & Measured $\sigma$ \\
\hline 0.1 & 0.102 & 0.003 \\
0.2 & 0.203 & 0.006 \\
0.3 & 0.305 & 0.010 \\
0.4 & 0.406 & 0.013 \\
0.5 & 0.507 & 0.016 \\
0.6 & 0.609 & 0.019 \\
0.7 & 0.709 & 0.021 \\
0.8 & 0.809 & 0.024 \\
0.9 & 0.912 & 0.028 \\
1.0 & 1.010 & 0.031 \\
1.1 & 1.111 & 0.034
\end{tabular}

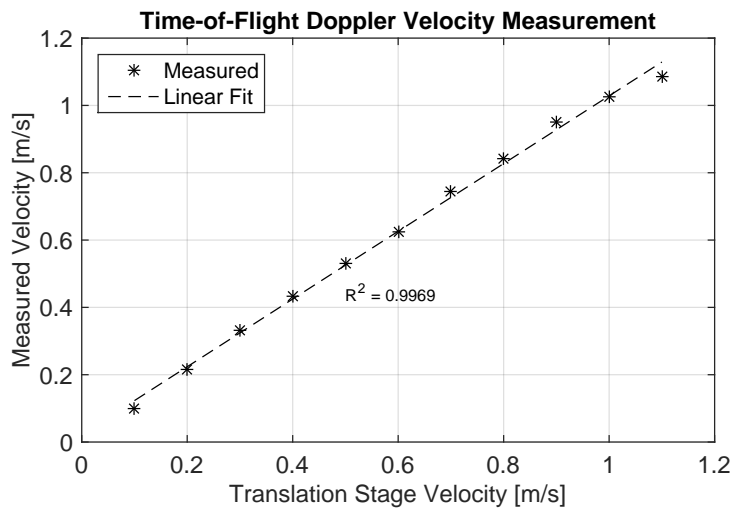

Fig. 3. Results of using ToF range cameras in Continuous Wave $(\mathrm{CW})$ mode to measure velocity.

same distance away from the camera. The mean and standard deviation of the region of pixels on the moving cardboard is tabulated in table 1 . The mean is within one standard deviation of the actual translation stage speed.

In Fig. 5 the velocity measurement is plotted at different times when the translation stage is moving at a velocity of $1.0 \mathrm{~m} / \mathrm{s}$. The velocity measurement of the cardboard sheet is maintained over the two second measurement window. The pixels on the edge of the cardboard sheet measure the velocity incorrectly because the motion in this region is a combination of radial and transverse motion due to the mixing of the foreground and background pixels.

Heide et al. [13] used heterodyne ToF to measure velocity. Herein we have adapted homodyne ToF camera technology which has the advantage of ease of implementation, especially in that homodyning does not require locking two frequencies. Unlike the work herein, Heide et al. provide a closed form solution for the computation of velocity [13]. They report errors less than $0.2 \mathrm{~m} / \mathrm{s}$, about twice the $99 \%$ margin of three times the largest standard deviation in Tab. 1. 


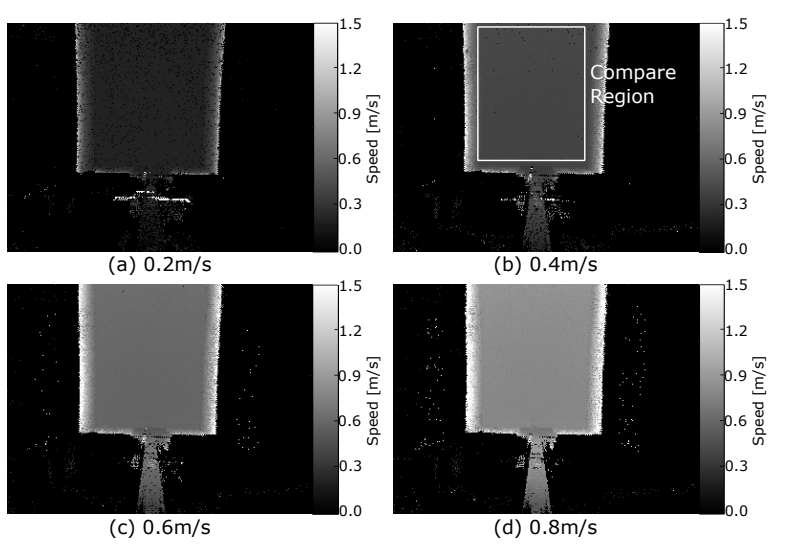

Fig. 4. CW velocity measurement results of moving cardboard sheet at the same distance away from the camera. In (a), (b), (c), and (d) the translation stage is moving at $0.2 \mathrm{~m} / \mathrm{s}, 0.4 \mathrm{~m} / \mathrm{s}$, $0.6 \mathrm{~m} / \mathrm{s}$ and $0.8 \mathrm{~m} / \mathrm{s}$ respectively. The mean and standard deviation of the pixels in the compare regions are tabulated in table 1 .

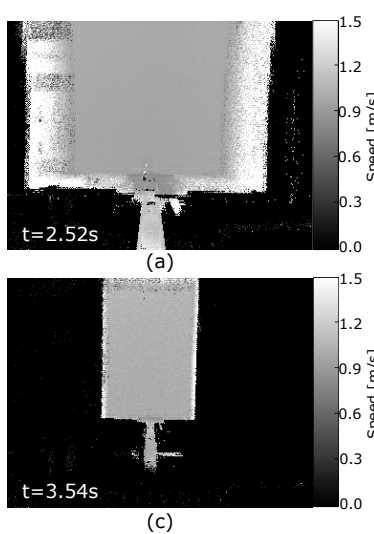

(c)

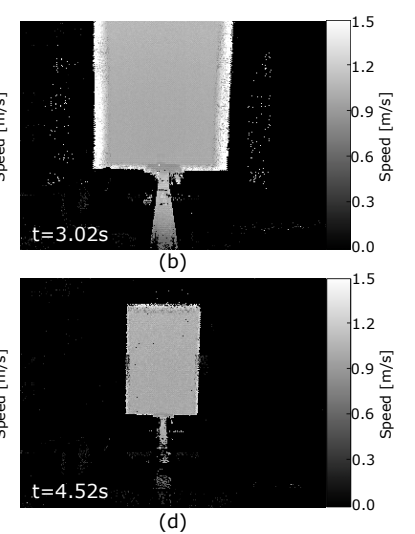

(d)
Fig. 5. CW velocity measurements for an object moving $2 \mathrm{~m}$ at $1.0 \mathrm{~m} / \mathrm{s}$ on a translation stage. The time of (a), (b), (c), and (d) is $2.52 \mathrm{~s}, 3.02 \mathrm{~s}, 3.54 \mathrm{~s}$, and $4.52 \mathrm{~s}$ respectively.

\section{B. Stepped Frequency Continuous Wave Modulation}

In this section the results for SFCW and AMCW modulation are compared with focus on the accuracy, phase wrapping and multi-path interference. The measured data when operating in SFCW mode for an object at $1.42 \mathrm{~m}$ and another object at $4.67 \mathrm{~m}$ is plotted in Fig. 6. Error bars indicate three standard deviations due to noise at the same pixel over 200 measurements. The more distant object has an estimated angular frequency of 1.269 radians and the closer object has an estimated angular frequency of 0.375 radians, which corresponds to $5.07 \mathrm{~m}$ and $1.50 \mathrm{~m}$ using Eqn. 21. In Fig. 7 we also summarise the raw SFCW data of 200 measurements of one pixel on the garden gnome's beard (just right of center in the images in Fig. 9) through the translucent sheet. In Fig. 7 we also show the reconstructed curve (solid line) found by substituting the estimated returns into Eqn. 23. The camera-gnome distance is approximately $3.25 \mathrm{~m}$. Two harmonics can be seen in the data, a low harmonic due to the foreground translucent sheet and a higher harmonic due to the gnome in the background. In Fig. 8 we show the MUSIC pseudo-spectrum of the data in Fig. 7. The two peaks correspond to the positions of the translucent sheet and gnome. We emphasise that this is not a 'true' spectrum in the Fourier sense because, while the peaks expose the presence of harmonics in the signal under analysis, the amplitude conveys no information about power of those harmonics.

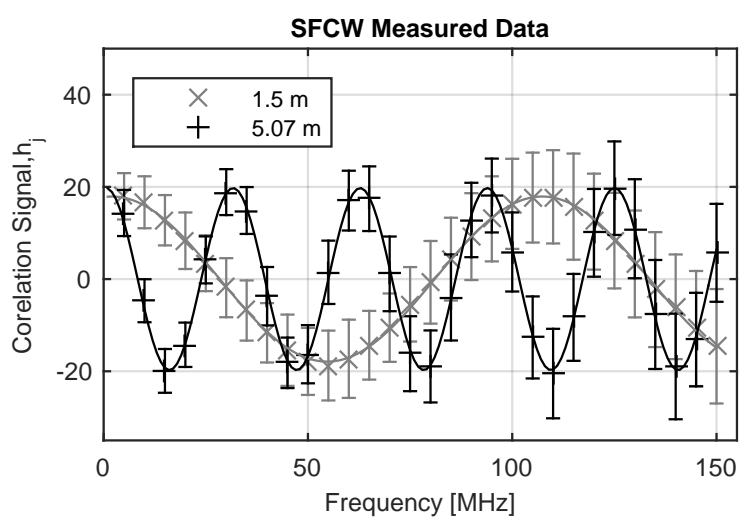

Fig. 6. Measured data from SFCW for objects at difference distances. The more distant object appears as a higher frequency correlation waveform signal than the closer object.

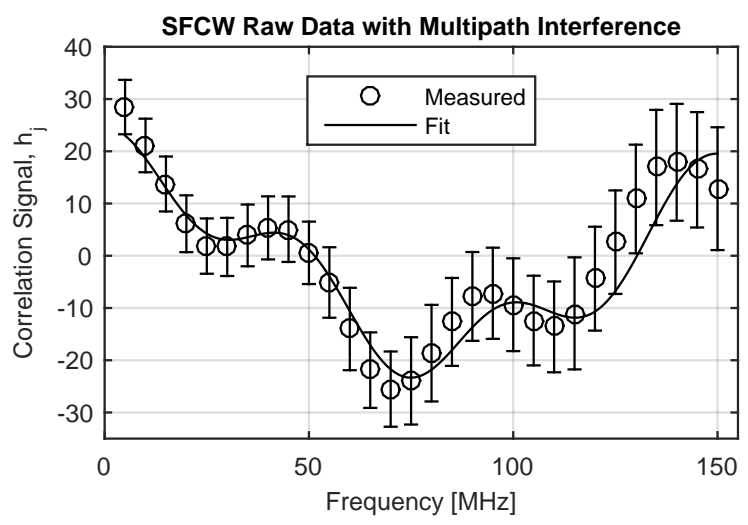

Fig. 7. Measured SFCW data with multi-path due to the translucent sheet. Error bars indicate three standard deviations due to noise at one pixel. The harmonics due to distance are apparent.

The comparison between AMCW and SFCW imaging the $8 \mathrm{~m}$ scene with and without a translucent sheet are in Fig. 9. A slice of distance from Fig. 9 is plotted in Figs. 10 and 11 comparing the AMCW and SFCW distance measurements. In the original scene the AMCW measurement suffers from phase wrapping, clearly visible in Fig. 10, while the SFCW measurement does not. There is a discrepancy at Columns 175-250 of Fig. 10 in the depth results between the SFCW and AMCW measurements. This discrepancy is believed to be caused by multi-path interference due to lens scattering of light from bright objects onto pixels that are directly imaging the dark curtain. The ground truth for the curtain is difficult to find, so we can only conjecture that the SFCW has corrected the lens scattering and leave conclusive assessment for future work. When the translucent sheet is present (Fig. 11) the AMCW amplitude and distance measurements are severely corrupted, and no usable depth is 


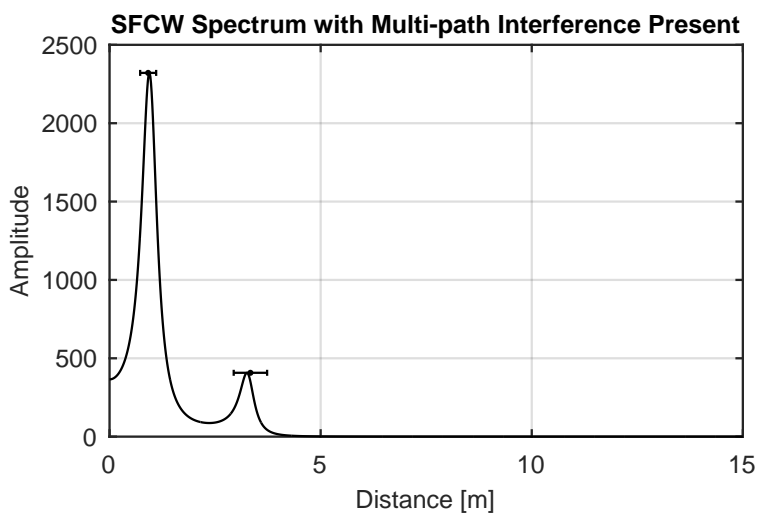

Fig. 8. Pseudo-spectrum analysis of the multi-path contaminated data in Fig. 7 by the MUSIC algorithm. The horizontal error bars show the three standard deviation variation of the location of the frequency peaks. The two returns are clearly separated.

measured. In contrast, when the SNR is sufficient, the SFCW measurements recover both the amplitude and distance behind the translucent sheet.

In Fig. 12 we show a comparison of distance measurements found for two values of $\Delta f$. For $\Delta f=15 \mathrm{MHz}, d_{a m g}=10 \mathrm{~m}$. However, the MUSIC method on real input data also limits the bandwidth of detected spectral components by another factor of two, so practically the ambiguity distance is reduced to $5 \mathrm{~m}$, and there is phase wrapping for the distance objects (Columns 0-60). For $\Delta f=5 \mathrm{MHz}$, there is no phase wrapping over the $8 \mathrm{~m}$ range in the test scene.

A box plot of the measured distance error of the pixels behind the translucent sheet for both AMCW and SFCW is in Fig. 13. The distance error is calculated from subtracting the distance between the measurements with, and without the translucent sheet. The box plot shows upper and lower quartiles; for AMCW the upper whisker is 1.5 times the interquartile range and the lower whisker is the lowest error values, and for SFCW the whiskers are both 1.5 times the interquartile range. The skewed shape of the AMCW error distribution is an artefact of this specific scene and may change for a different scene. Referring to table 2, the SFCW measurements have much lower median error than $\mathrm{AMCW}$, and the spread in error as measured by the interquartile range is reduced by 22 times. This result demonstrates that for a typical scene SFCW measurements are robust to multi-path interference that plagues AMCW operation.

Table 2. Quartiles of distance errors through a translucent sheet.

\begin{tabular}{c|cc} 
Quartile & AMCW & SFCW \\
\hline 25 & $-4.0 \mathrm{~m}$ & $-0.18 \mathrm{~m}$ \\
50 & $-1.3 \mathrm{~m}$ & $-0.06 \mathrm{~m}$ \\
75 & $0.0 \mathrm{~m}$ & $0.0 \mathrm{~m}$
\end{tabular}

The accuracy between AMCW and SFCW over $1.05 \mathrm{~m}$ is plotted in Fig. 14, comparing SFCW using 10 frequency steps (and other numbers of frequency steps, discussed below) with AMCW at $50 \mathrm{MHz}$ using 9 phase steps. The error bars show one standard deviation of the measurement. The SFCW mea-

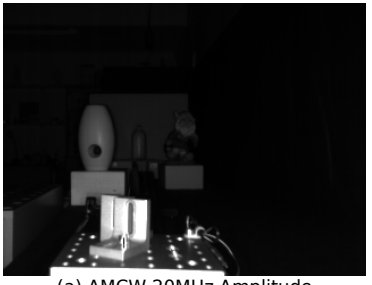

(a) AMCW $20 \mathrm{MHz}$ Amplitude

(c) AMCW $20 \mathrm{MHz}$ Amplitude

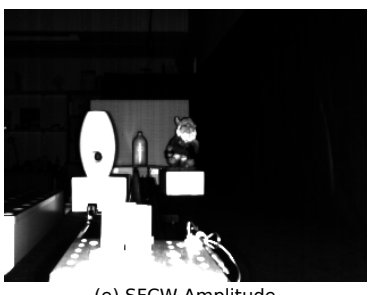

(e) SFCW Amplitude

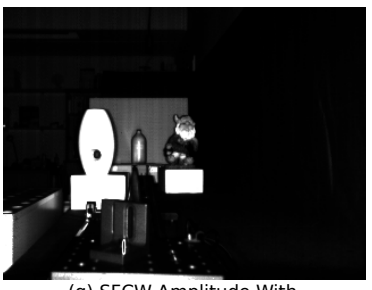
(g) SFCW Amplitude With
Translucent Sheet

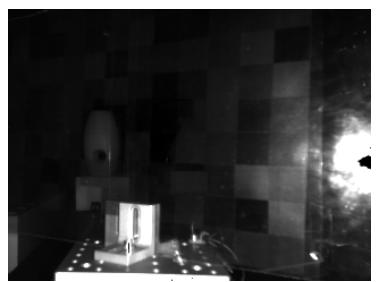

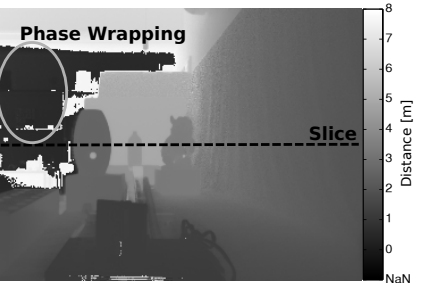

(b) AMCW $20 \mathrm{MHz}$ Distance

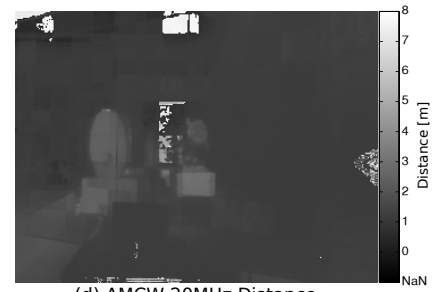

(d) AMCW 20MHz Distance
With Translucent Sheet

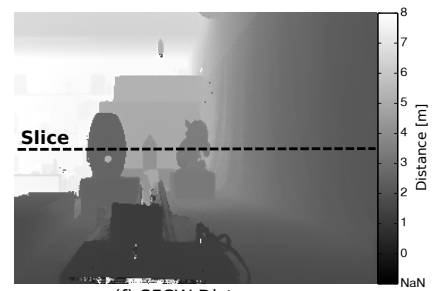

(f) SFCW Distance

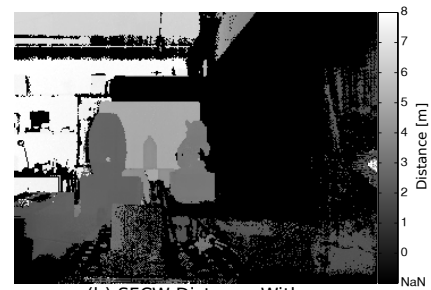

(h) SFCW Distance With
Translucent Sheet
Fig. 9. Comparison between AMCW measurements in (a), (b), without a translucent sheet and (c), (d) with a translucent sheet. These are compared to SFCW measurements in (e), (f) without a translucent sheet and (g), (h) with a translucent sheet. Comparison of distance slices is in Figs. 10 and 11.

surement oscillates around zero with an peak error of $0.02 \mathrm{~m}$, compared to the AMCW where the maximum error is $0.005 \mathrm{~m}$. Beyond $1.2 \mathrm{~m}$ there is overlap in the accuracy to one standard deviation of noise between AMCW and SFCW using 10 frequency steps. The SFCW distance measurement using the MUSIC method is significantly improved over using the DFT to resolve distance. With the DFT the minimum resolvable distance with a bandwidth of $150 \mathrm{MHz}$ using Eqn. 22 is $1 \mathrm{~m}$. The MUSIC spectrum estimation method is able to decrease the minimum resolvable distance, but is not perfect. As the bandwidth of ToF range cameras improves the accuracy will improve linearly with bandwidth.

The accuracy of SFCW with the number of frequency steps is also plotted in Fig. 14, comparing the accuracy with 6, 10 and 30 samples. To one standard deviation, there is no separation between 10 and 30 samples. With more frequency samples the error decreases, therefore there is a trade off between accuracy and frame rate. Currently most ToF cameras use four measurements to compute depth, therefore six is realistic for $30 \mathrm{fps}$ of depth information, making it possible to implement SFCW in real-time. 


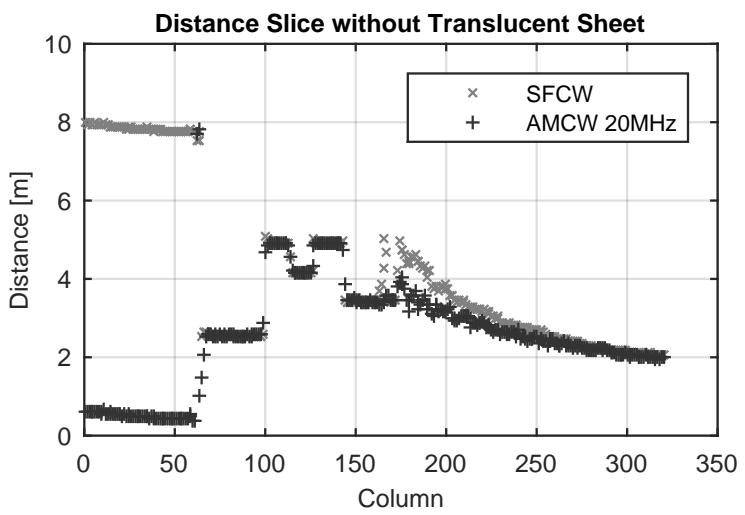

Fig. 10. Distance slice comparing AMCW and SFCW without a translucent sheet. The AMCW suffers from phase wrapping and multi-path interference, while the SFCW does not.

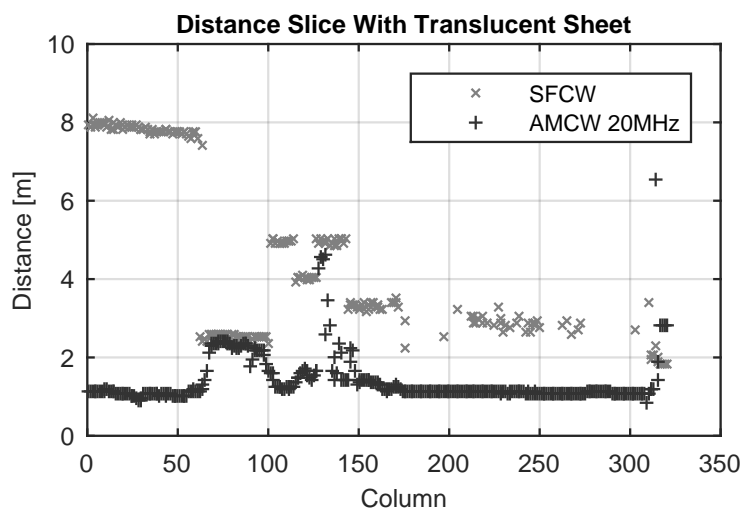

Fig. 11. Distance slice comparing AMCW and SFCW with a translucent sheet present. The multi-path interference from the translucent sheet pulls the AMCW measurements closer to the camera, whereas SFCW is resistant to the multi-path interference.

The application of lidar techniques to AMCW ToF cameras is possible because of the advances of the ToF imaging arrays, the maximum modulation frequency has increased significantly over the last ten years [4]. The closest previous work using ToF range cameras with multiple modulation frequencies is Kirmani [15], who take complex measurements at five frequencies and resolving multi-path interference with spectrum estimation and phase unwrapping the data. Sampling over five frequencies requires a minimum of 15 raw frames, and this is difficult to achieve when $30 \mathrm{fps}$ of depth information is required. A disparate example to the work herein [18] used hundreds of frequencies and stepped the phase for full transient imaging, a problem that is more general than the correction of multi-path. The ideas in [18], while powerful and practical for analysis of light transport, are not suitable for real-time imaging. In contrast to both [15] and [18], the work herein does not use phase stepping, but instead relies only on multi-frequency data acquisition. In this paper we show that multi-frequency techniques are the equivalent of SFCW. In SFCW mode the depth can be measured with a similar number of measurements as AMCW, potentially allowing for $30 \mathrm{fps}$ of depth information AMCW ToF range cameras suffer from scene dependent measurement errors caused by phase wrapping and multi-path interference. The results

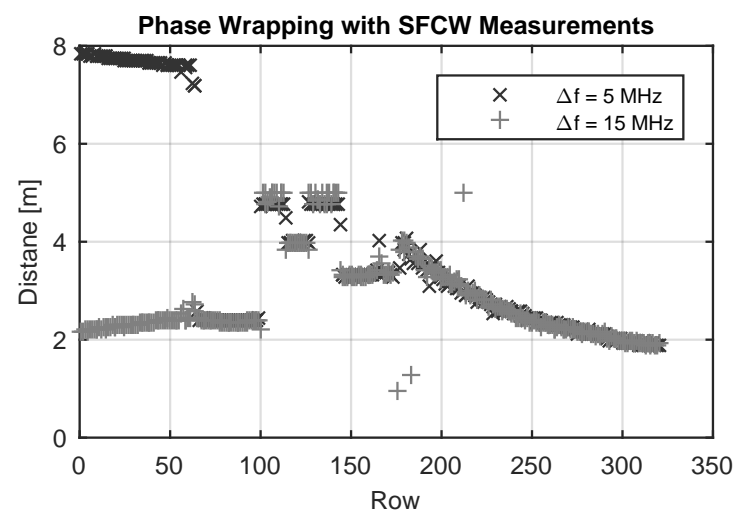

Fig. 12. Phase wrapping with frequency step size. Increasing the step size reduces the ambiguity distance.

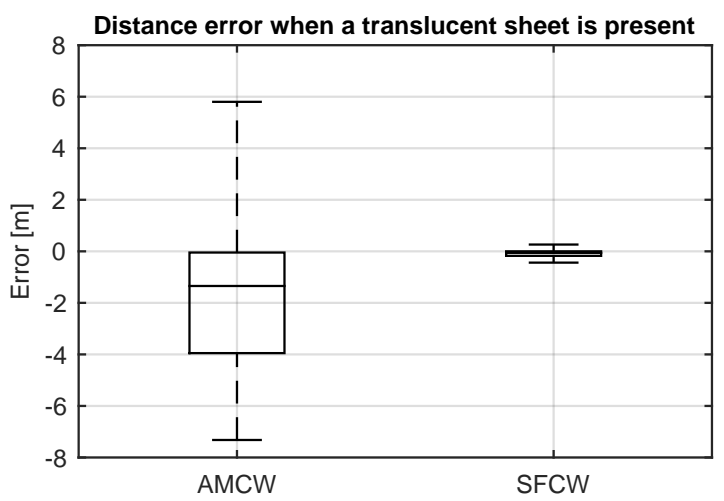

Fig. 13. Box plot of the distance measurement error of pixels behind a translucent sheet for AMCW and SFCW measurement techniques. The translucent sheet causes much less multi-path error in SFCW than AMCW.

show that the accuracy of SFCW is resilient to phase wrapping and multi-path interference. However, the accuracy of SFCW accuracy is dependent on the frequency bandwidth. If the trend of higher operating frequencies carries on then the accuracy of operating in SFCW mode will improve, making operating in SFCW increasingly attractive.

In future work other Radar techniques such as synthetic aperture radar and beam forming can also be employed. There has been work on applying compressed sensing techniques to SFCW ground penetrating radar to reduce the number of frequency measurements. These techniques could easily be applied to ToF range cameras.

\section{CONCLUSION}

Techniques from radar and lidar imaging have been applied to time-of-flight (ToF) range cameras. SFCW is a measurement technique that is resistant to the measurement errors that plague current ACMW ToF range cameras. It is resistant to multi-path interference, phase wrapping and harmonic aliasing. However the accuracy of the measurement is proportional to the frequency bandwidth. Both CW and SFCW can potentially be performed at standard video rates. $\mathrm{CW}$ has been applied to ToF cameras to measure the velocity of moving objects. As the modulation frequency bandwidths increase in ToF range cameras SFCW will be more advantageous. 


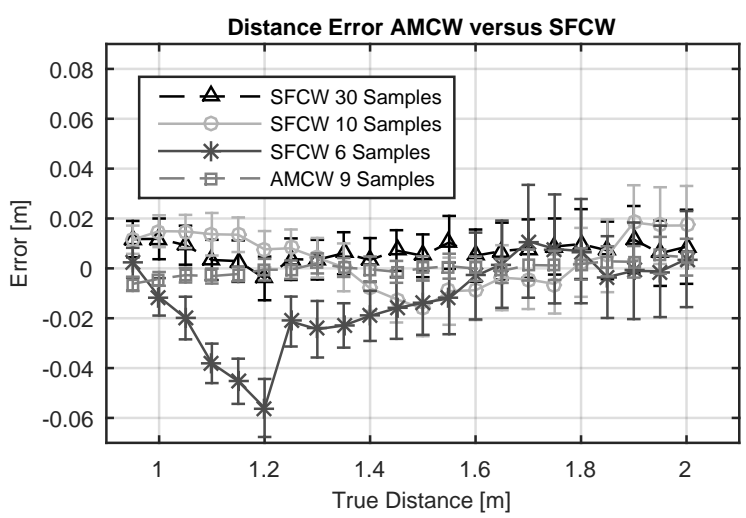

Fig. 14. Accuracy of SFCW distance measurements over the number of samples used, and compared to AMCW of measurement, each without multi-path. For 10 and 30 samples, below $1.2 \mathrm{~m}$ SFCW has greater error than AMCW. Above $1.2 \mathrm{~m}$ the measurements overlap to one standard deviation of noise. For 6 samples SFCW overlaps with AMCW at $1.4 \mathrm{~m}$

\section{REFERENCES}

1. J. P. Godbaz, M. J. Cree, and A. A. Dorrington, "Understanding and ameliorating non-linear phase and amplitude responses in amcw lidar," Remote Sensing 4, 21-42 (2011).

2. Z. W. Barber, J. R. Dahl, A. B. Mateo, S. Crouch, and R. Reibel, "High resolution FMCW ladar for imaging and metrology," in "Imaging and Applied Optics, OSA Technical Digest (online)," (2015), p. LM4F.2.

3. F. Heide, L. Xiao, A. Kolb, M. B. Hullin, and W. Heidrich, "Imaging in scattering media using correlation image sensors and sparse convolutional coding," Optics Express 22, 26338-26350 (2014).

4. C. Bamji, P. O'Connor, T. Elkhatib, S. Mehta, B. Thompson, L. Prather, D. Snow, O. Akkaya, A. Daniel, A. Payne, T. Perry, M. Fenton, and V.-H. Chan, "A $0.13 \mu \mathrm{m}$ CMOS system-on-chip for a $512 \times 424$ time-of-flight image sensor with multi-frequency photo-demodulation up to $130 \mathrm{MHz}$ and 2 GS/s adc," Solid-State Circuits, IEEE Journal of 50, 303-319 (2015).

5. A. D. Payne, A. A. Dorrington, M. J. Cree, and D. A. Carnegie, "Improved measurement linearity and precision for AMCW time-of-flight range imaging cameras," Applied Optics 49, 2392-4403 (2010).

6. R. Crabb and R. Manduchi, "Probabilistic phase unwrapping for singlefrequency time-of-flight range cameras," in "Proceeding of the International Conference on 3D Vision (3DV 14)," (Tokyo, Japan, 2014).

7. L. Streeter and A. A. Dorrington, "Coded exposure correction of transverse motion in full-field range imaging," Optical Engineering 53, 102109-1-102109-10 (2014).

8. M. Lindner and A. Kolb, "Compensation of motion artifacts for time-offlight cameras," in "Dynamic 3D Imaging," , vol. 5742 (2009), vol. 5742, pp. $16-27$.

9. A. A. Dorrington, M. J. Cree, A. D. Payne, R. M. Conroy, and D. A. Carnegie, "Achieving sub-millimetre precision with a solid-state fullfield heterodyning range imaging camera," Measurement Science and Technology 18, 2809-2816 (2007).

10. P. Gulden, M. Vossiek, P. Heide, and R. Schwarte, "Novel opportunities for optical level gauging and 3D-imaging with the photoelectronic mixing device," Instrumentation and Measurement, IEEE Transactions on 51, 679-684 (2002).

11. A. Kolb, E. Barth, R. Koch, and R. Larsen, "time-of-flight sensors in computer graphics," Computer Graphics Forum 29, 141-159 (2010).

12. M. Lindner, A. Kolb, and T. Ringbeck, "New insights into the calibration of ToF-sensors," in "IEEE Computer Society Conference on Computer Vision and Pattern Recognition Workshops (CVPRW'08)," (Anchorage, Alaska, USA, 2008), pp. 1-5.

13. F. Heide, W. Heidrich, M. Hullin, and G. Wetzstein, "Doppler time-offlight imaging," ACM Transactions on Graphics 34, 36:1-36:11 (2015).
14. D. Freedman, Y. Smolin, E. Krupka, I. Leichter, and M. Schmidt, "SRA: fast removal of general multipath for ToF sensors," in "European Conference on Computer Vision (ECCV)," (Zurich, Switzerland, 2014), pp. 234-249.

15. A. Kirmani, A. Benedetti, and P. A. Chou, "SPUMIC: Simultaneous phase unwrapping and multipath interference cancellation in time-offlight cameras using spectral methods," in "IEEE International Conference on Multimedia and Expo (ICME)," (IEEE, 2013), pp. 1-6.

16. D. Droeschel, D. Holz, and S. Behnke, "Multi-frequency phase unwrapping for time-of-flight cameras," in "2010 IEEE/RSJ International Conference on Intelligent Robots and Systems (IROS)," (Taipei, Taiwan, 2010), pp. 1463-1469.

17. A. Kadambi, , R. Whyte, A. Bhandari, L. Streeter, C. Barsi, A. Dorrington, and R. Raskar, "Coded time of flight cameras: sparse deconvolution to address multipath interference and recover time profiles," ACM Transactions on Graphics 32, 167:1-167:10 (2013).

18. F. Heide, M. B. Hullin, J. Gregson, and W. Heidrich, "Low-budget transient imaging using photonic mixer devices," ACM Transactions on Graphics 32, 45:1-45:10 (2013).

19. A. A. Dorrington, J. P. Godbaz, M. J. Cree, A. D. Payne, and L. V. Streeter, "Separating true range measurements from multi-path and scattering interference in commercial range cameras," Proc. SPIE 7864, 786404 (2011)

20. A. Bhandari, A. Kadambi, R. Whyte, C. Barsi, M. Feigin, A. Dorrington, and R. Raskar, "Resolving multipath interference in time-of-flight imaging via modulation frequency diversity and sparse regularization," Optics Letters 39, 1705-1708 (2014).

21. J. Lin, Y. Liu, M. B. Hullin, and Q. Dai, "Fourier analysis on transient imaging by multifrequency time-of-flight camera," in "IEEE Conference on Computer Vision and Pattern Recognition (CVPR)," (Columbus, Ohio, USA, 2014), pp. 3230-3237.

22. G. L. Charvat, Small and Short-Range Radar Systems (CRC Press, 6000 Broken Sound Parkway NW, Suite 300 Boca Raton, FL 33487, 2014).

23. C. J. Karlsson, F. A. A. Olsson, D. Letalick, and M. Harris, "All-fiber multifunction continuous-wave coherent laser radar at $1.55 \mu \mathrm{m}$ for range, speed, vibration, and wind measurements," Applied Optics 39, 3716-3726 (2000).

24. M. Skolnik, Radar Handbook, Electronics electrical engineering (McGraw-Hill Education, 2008), 3rd ed.

25. M. L. Simpson, M. Cheng, T. Q. Dam, K. E. Lenox, J. R. Price, J. M. Storey, E. A. Wachter, and W. G. Fisher, "Intensity-modulated, stepped frequency $\mathrm{CW}$ lidar for distributed aerosol and hard target measurements," Applied Optics 44, 7210-7217 (2005).

26. A. D. Payne, A. A. Dorrington, M. J. Cree, and D. A. Carnegie, "Characterization of modulated time-of-flight range image sensors," Proc. SPIE 7239, 723901 (2008).

27. R. Lange, "3D time-of-flight distance measurement with custom solidstate image sensors in CMOS/CCD technology," Ph.D. thesis, University of Siegen (2000).

28. A. D. Payne, A. P. P. Jongenelen, A. A. Dorrington, M. J. Cree, and D. A. Carnegie, "Multiple frequency range imaging to remove measurement ambiguity," in "9th Conference on Optical 3-D Measurement Techniques," (Vienna, Austria, 2009), pp. 1-3.

29. J. Seiter, , M. Hofbauer, M. Davidovic, and H. Zimmermann, "Correction of a phase dependent error in a time-of-flight range sensor," Proc. SPIE 8791, 87910 (2013)

30. R. Whyte, L. Streeter, M. J. Cree, and A. A. Dorrington, "Review of methods for resolving multi-path interference in time-of-flight range cameras," in "IEEE Sensors," (Valencia, Spain, 2014), pp. 629-632.

31. D. J. Daniels, Ground penetrating radar (Wiley Online Library, 2005).

32. S. M. Kay and S. L. M. Jr, "Spectrum analysis a modern perspective," Proceedings of the IEEE 69, 1380-1419 (1981).

33. P. Stoica and R. L. Moses, Introduction to spectral analysis, vol. 1 (Prentice hall Upper Saddle River, 1997). 